\title{
Use of intraabdominal VAC (Vacuum Assisted Closure) lowers mortality and morbidity in patients with open abdomen
}

\author{
Hutan M Jr${ }^{1}$, Hutan M Sen ${ }^{2}$, Skultety $\mathrm{J}^{1}$, Sekac J ${ }^{1}$, Koudelka $\mathrm{P}^{1}$, Prochotsky $\mathrm{A}^{1}$, Yaghi A ${ }^{3}$, Labas $\mathrm{P}^{4}$ \\ 2nd Surgical Clinic of School of Medicine Comenius University, University Hospital Bratislava, Hospital St Cyril and Methodius, \\ Bratislava, Slovakia. matohuto@yahoo.com
}

\begin{abstract}
Objective: Authors compare two groups of patients with open abdomen. The objective is to compare and evaluate two treatment modalities, namely Kern laparostomy and vacuum-assisted closure in terms of mortality, closure of abdominal wound, and fistula management, all these stratified by BMI and CRP.

Background: Open abdomen can be considered a "patient salvage technique", used in patients with abdominal sepsis, as well as in patients with abdominal compartment syndrome, and in damage control surgery. Various management techniques are known, of which Kern laparostomy is most widely used. Newer techniques using negative pressure have emerged, still waiting for their wider acceptance and use. The authors present their study, in which they compare Kern laparostomy and intraabdominal VAC in patients with open abdomen. Material and methods: Study consists of 44 patients treated at the authors' clinics, while group KERN consisted of patients managed by Kern laparostomy, and group VAC was managed by intraabdominal VAC. The groups were compared in terms of mortality, abdominal closure, appearance of enteroatmospheric fistulas, primary closure of fistulas, and possibility of diversion of enteral contents. All outputs were stratified by CRP (C-reactive protein) and BMI (Body Mass Index).

Results: In VAC group, a significant decrease in mortality was seen, as well as significantly higher closure of abdominal wall, and significantly higher possibility of diversion of enteral content from fistulas. No statistically significant findings were observed in stratification with CRP and BMI.

Conclusion: Intraabdominal VAC offers patients lower morbidity and mortality and should be defined as a treatment of choice in patients with open abdomen (Tab. 4, Fig. 3, Ref. 15). Full Text in PDF www.elis.sk.

Key words: VAC, open abdomen, Kern laparostomy, SMAC, dynamic compression sutures.
\end{abstract}

Open abdomen (OA, laparostomy) is a rather new method of managing complicated intraabdominal pathologies, and can be considered a ,patient salvage therapy“. The management of patients with $\mathrm{OA}$ is complicated, challenging and frustrating. Close interdisciplinary cooperation of surgeons, anaesthesiologists, radiologists and pharmacologists are needed, while the outcomes are hardly predictable and the morbidity and mortality rates are high. There are many possibilities of local OA management; the most commonly used method is Kern laparostomy employing a transparent adhesive drape for covering the laparostomy (1). The introduction of VAC (Vacuum Assisted Closure) with its intraabdominal application into the management of complicated wounds showed

\footnotetext{
2nd Surgical Clinic of School of Medicine Comenius University, University Hospital Bratislava, Hospital St Cyril and Methodius, Bratislava, ${ }^{2} 4$ th Surgical Clinic of School of Medicine Comenius University, University Hospital Bratislava, Hospital Ruzinov, Bratislava, ${ }^{3}$ Anaesthesiology and Intensive Medicine Clinic, University Hospital Bratislava, Hospital Ruzinov, Bratislava, and ${ }^{4} 1$ st Surgical Clinic of School of Medicine Comenius University, University Hospital Bratislava - Hospital Stare Mesto, Bratislava, Slovakia
}

Address for correspondence: M. Hutan, MD, 2nd Surgical Clinic of School of Medicine Comenius University, University Hospital Bratislava, Hospital St Cyril and Methodius, Antolska 11, SK-851 07 Bratislava, Slovakia.

Acknowledgement: This work was created without any grant support and authors have no conflicts of interest. promising results $(2,3,4)$. Our aim was to compare two groups of patients with OA, namely one managed by Kern laparostomy, the other by intraabdominal VAC, and to statistically evaluate their outcomes in terms of mortality, closure of abdominal cavity, incidence of enteroatmospheric fistulas, possibility of their primary closure, as well as the possibility of diverting the enteral content from fistulas. All these outcomes were stratified with maximum CRP (C-reactive protein) as a marker of inflammation, and BMI (Body Mass Index) as that of patient's constitution.

\section{Material and methods}

Patients included in the study come from four clinical wards, IVth Surgical Clinic of University Hospital Bratislava (20002011), IInd Surgical Clinic of University Hospital Bratislava (2006-2011), Clinic of Anaesthesiology and Intensive Care of University Hospital Bratislava (2000-2011), and Department of Anaesthesiology and Intensive Care of University Hospital Bratislava - Hospital St. Cyril and Methodius (2006-2011). Until 2006, all patients were managed by Kern laparostomy, and we identified and evaluated them retrospectively. This group, referred to as KERN, consists of 17 patients ( 9 males, 8 females, mean age 51.06 years). From 2007 onward, we started to evaluate all patients with OA prospectively, manage them with intraabdominal VAC and refer to this group as VAC. It consisted of 27 patients (13 males, 14 fe- 
Tab. 1. Indications for open abdomen.

\begin{tabular}{lcccccccc}
\hline & \multicolumn{2}{c}{ KERN } & & \multicolumn{2}{c}{ VAC } & & \multicolumn{2}{c}{ KERN+VAC } \\
\cline { 2 - 3 } \cline { 8 - 9 } & $\mathrm{n}$ & $\%$ & & $\mathrm{n}$ & $\%$ & & $\mathrm{n}$ & $\%$ \\
\hline 1. ACS & 5 & $29,41 \%$ & & 8 & $29,63 \%$ & & 13 & $29,55 \%$ \\
2. TP & 8 & $47,06 \%$ & & 12 & $44,44 \%$ & & 20 & $45,45 \%$ \\
3. DCS & 3 & $17,65 \%$ & & 3 & $11,11 \%$ & & 6 & $13,64 \%$ \\
4. DL & 1 & $5,88 \%$ & & 4 & $14,81 \%$ & & 5 & $11,36 \%$ \\
\hline sum & 17 & $100,00 \%$ & & 27 & $100,00 \%$ & & 44 & $100,00 \%$ \\
\hline
\end{tabular}

ACS - Abdominal Compartment Syndrome

$\mathrm{TP}$ - tercial peritonitis

DCS - damage control surgery

$\mathrm{DL}$ - dehiscence of laparotomy

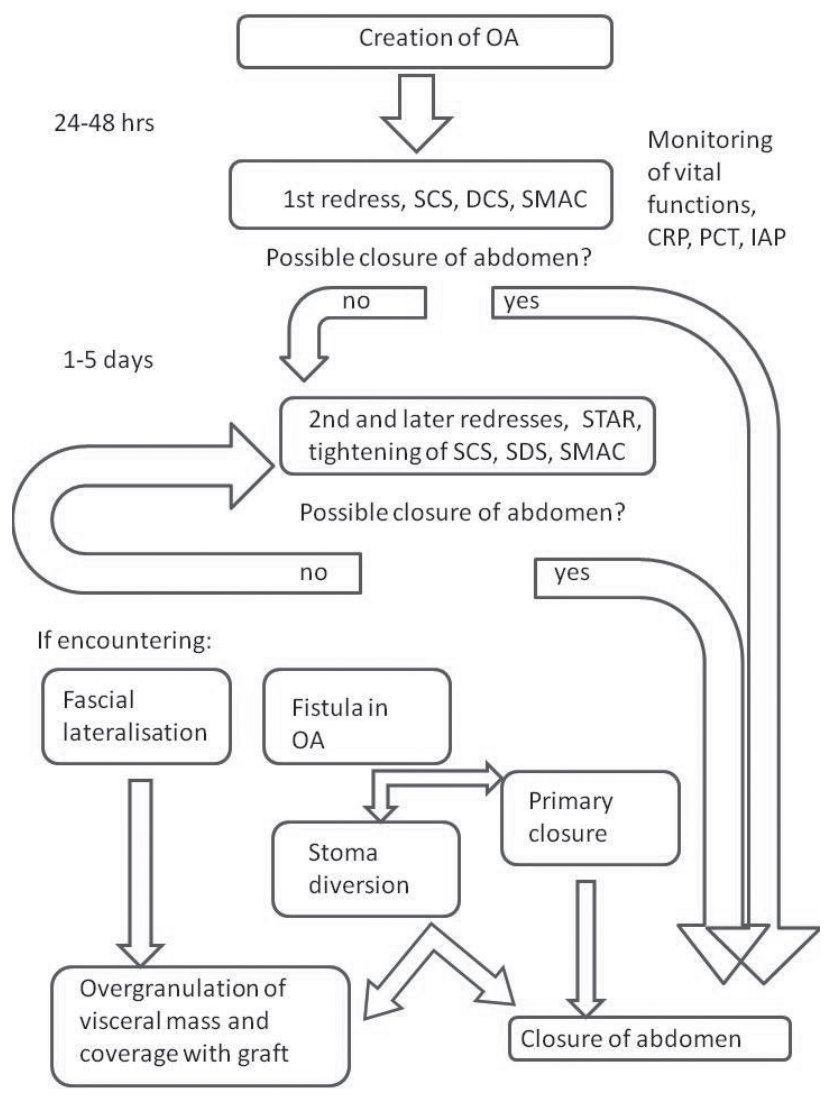

Fig. 1. Algorithm of use of intraabdominal VAC.

males, mean age 55.96 years). In total, both groups consisted of 44 patients ( 22 males, 22 females, mean age 54.07 years).

Indications for opening the abdomen are in Table 1. In patients with Kern laparostomy, a transparent 3M drape (3M, St. Paul. MN, USA) was used for covering the laparostomy with or without gauze drapes. Redressing of the wound was done daily, usually at the operating theatre. In case of regression of oedema and enhancement of clinical state, resuture of the abdominal wall was attempted. If this was not possible, laparostomy was left to heal by means of granulation tissue and this was later covered by split-skin graft. In patients with VAC we adhered to Figure 1. In first patients, we started with simple intraabdominal VAC, later we combined it with static compression sutures (SCS), staged ab- dominal repair (STAR), dynamic compression sutures (DCS), and even SMAC (Sandwich Mesh Abdominal Closure) (5). We used ATS KCI (Clinical Concepts Incorporated., San Antonio, Texas, USA) with Granufoam in OA in absence of fistulas and in combination with Whitefoam in OA in presence of fistulas. Redresses were done every 3-4 days, depending on the clinical state of the patient, i.e. the closure was done when considered safe, if not, overgranulation and coverage with skin graft was used.

Fistula management in KERN group was done by simple absorbable sutures. In cases that the primary suture had shown to be unsuccessful, the techniques such as "floating stoma" or pertubation with Petzer catheter almost completely failed to divert the enteral content. In VAC group, the fistula was managed with use of Whitefoam. As long as the mouth of fistula was no bigger than $0.5 \mathrm{~cm}$, no eversion of mucosa was seen. If this was not the case or the primary closure was not successful, we tried to divert the enteral content from abdominal cavity by "fistula VAC" sec. Govermann (6).

After gathering the data, these were summarized with help of Microsoft Excel 2007, (C2006 (Microsoft Corporation, Redmond, WA, USA). All results were statistically evaluated by GraphPad Prism 5, C 1992-2010 ( GraphPad Software, San Diego, CA, USA.)

\section{Results}

Primary endpoints of the study in terms of hospitalisation time, mortality, and closure of laparostomy were on days 7, 10 and 30. The results are seen in Tables 2 and 3. The mortality in VAC group was lower than that in KERN group, namely by 27.64 $\%(p=0.0256)$, and the rate of abdominal closure in the former group was higher by $35.95 \%(\mathrm{p}=0.0397$, statistically evaluated with Log-Rank (Mantel-Cox) test).

Tab. 2. Evaluation of mortality after creation of $\mathrm{OA}$.

\begin{tabular}{|c|c|c|c|c|c|c|}
\hline & \multicolumn{2}{|c|}{ KERN } & \multicolumn{2}{|c|}{ VAC } & \multicolumn{2}{|c|}{ KERN+VAC } \\
\hline & $\mathrm{n}$ & $\%$ & $\mathrm{n}$ & $\%$ & $\mathrm{n}$ & $\%$ \\
\hline$\overline{\mathrm{A}}$ & 1 & $5,88 \%$ & 0 & $0,00 \%$ & 1 & $2,27 \%$ \\
\hline B & 2 & $11,76 \%$ & 0 & $0,00 \%$ & 2 & $4,55 \%$ \\
\hline $\mathrm{C}$ & 5 & $29,41 \%$ & 1 & $3,70 \%$ & 6 & $13,64 \%$ \\
\hline D & 11 & $64,71 \%$ & 10 & $37,04 \%$ & 21 & $47,73 \%$ \\
\hline
\end{tabular}

$\mathrm{p}<0,05$ for total mortality

A - mortality to 7 days after creation of OA

$\mathrm{B}$ - mortality to 10 days after creation of OA

$\mathrm{C}$ - mortality to 30 days after creation of OA

$\mathrm{D}$ - mortality until end of hospitalization for primary diagnosis

Tab. 3. Closure of OA.

\begin{tabular}{ccccccccc}
\hline & \multicolumn{2}{c}{ KERN } & & \multicolumn{2}{c}{ VAC } & & \multicolumn{2}{c}{ KERN+VAC } \\
\cline { 2 - 3 } \cline { 8 - 9 } \cline { 8 - 9 } & $\mathrm{n}$ & $\%$ & & $\mathrm{n}$ & $\%$ & & $\mathrm{n}$ & $\%$ \\
\hline $\mathrm{A}$ & 2 & 11,76 & & 0 & - & & 2 & 4,55 \\
$\mathrm{~B}$ & 3 & 17,65 & & 3 & 11,11 & & 6 & 13,64 \\
$\mathrm{C}$ & 7 & 41,18 & & 19 & 70,37 & & 26 & 59,09 \\
$\mathrm{D}$ & 9 & 52,94 & & 24 & 88,89 & & 33 & 75,00 \\
\hline
\end{tabular}

$\mathrm{p}<0,05$ closure during hospitalization

A - closure of abdomen up to 7 days after creation of OA

$\mathrm{B}$ - closure of abdomen up to 10 days after creation of OA

$\mathrm{C}$ - closure of abdomen up to 30 days after creation of OA

$\mathrm{D}$ - closure od abdomen until end of hospitalization for primary diagnosis 
Tab. 4. Assessment of fistula management in OA.

\begin{tabular}{ccccccccc}
\hline & \multicolumn{2}{c}{ KERN } & & \multicolumn{2}{c}{ VAC } & & \multicolumn{2}{c}{ KERN+VAC } \\
\cline { 2 - 3 } \cline { 8 - 9 } & $\mathrm{n}$ & $\%$ & & $\mathrm{n}$ & $\%$ & & $\mathrm{n}$ & $\%$ \\
\hline $\mathrm{A}$ & 4 & $23,5 \%$ & & 9 & $33,3 \%$ & & 13 & $29,5 \%$ \\
$\mathrm{~B}$ & 0 & $0 \%$ & & 1 & $11,1 \%$ & & 1 & $7,7 \%$ \\
$\mathrm{C}$ & 0 & $0 \%$ & & 6 & $66,7 \%$ & & 6 & $46,2 \%$ \\
\hline
\end{tabular}

$\mathrm{p}<0,05$ for fistula diversion

$\mathrm{A}$ - fistula incidence

B - successful fistula closure

$\mathrm{C}$ - successful fistula diversion

Secondary endpoints included the incidence of enteroatmospheric fistula in OA, primary closure of fistula, and possibility of diverting the enteral contents outside the opened abdomen. The results are seen in Table 4. Successful fistula diversion was significantly higher in VAC group ( $\mathrm{p}=0.0325$, Log-Rank (MantelCox) test).

Primary and secondary endpoints were stratified with highest CRP (mg/l) and BMI $\left(\mathrm{kg} / \mathrm{m}^{2}\right)$. No statistically significant differences were found.

\section{Discussion}

The management of OA is long, frustrating and its outcome is dubious. The treatment of abdominal sepsis consists of three parts, particularly surgical sanitation of infection, antibiotic therapy, and organ support. OA management helps in dealing with the causes by surgical debridement and cleaning the source area of infection, as well as in dealing with consequences of infection in open abdomen (abdominal compartment syndrome and its decompression).

Patients with OA form a quite diverse group of patients with distinct primary and associated diseases. At the same time their numbers are low, and even in high-volume centres, their numbers reach 5-10 per year. Having in mind such differences in their illnesses, the assessment and statistical evaluation of such patients is very difficult. In our study, the composition of patients is very similar in terms of most common underlying diseases. In KERN group, more patients were managed by damage control surgery

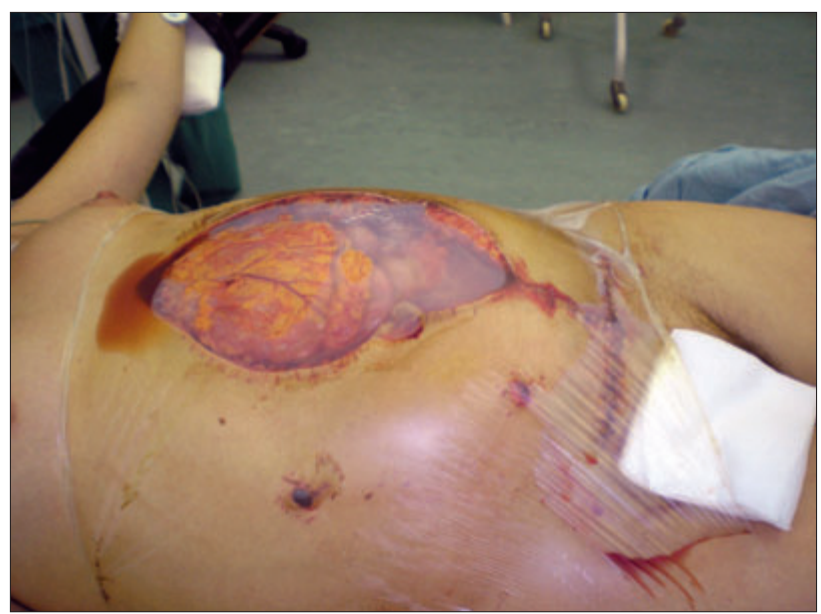

Fig. 2. Patient with Kern laparostomy (Patient after HELLP syndrome, note wound after Caesarean section).
(17.65 \% vs $11.11 \%$ ), while in VAC group more patients developed laparotomic wound dehiscence (14.81\% vs $5.88 \%$ ). Mean age of VAC group is higher by almost 5 years ( 55.96 vs $51.06 \mathrm{yrs}$.), mean BMI in VAC group is higher by $3 \mathrm{~kg} / \mathrm{m}^{2}\left(29.12 \mathrm{vs} 26.07 \mathrm{~kg} / \mathrm{m}^{2}\right)$.

Even though we have specific guidelines for the management of underlying disease, we still lack guidelines for that of OA. As from 2009, we are using a scoring system for open abdomen (7).

There are very few treatment options for OA, and Kern laparostomy has been used in all clinics since first cases. We have not used Wittman patch or Ethizip in any of the patients. Based on the resemblance of closure types, we presume that the results would be similar. Because of specific aspects such as loss of domain formation, fascial lateralisation, complicated fistula management and high septic morbidity of patients, we started using intraabdominal VAC instead, and this despite that no evidence-based studies for its intraabdominal use had been published at that time. As the need for these heightened, we retrospectively evaluated the patients with Kern laparostomy and started to evaluate prospectively those with VAC. With advances in wound treatment over time, we included static and dynamic sutures, STAR and SMAC into therapy.

In terms of mortality, we proved that the use of intraabdominal VAC significantly lowers mortality. The biggest task is probably done by active drawing off the bacterial load and their metabolites, promotion of granulation and quicker closure. In Table 2, a slower accrual of mortality is seen, ending up by $27.64 \%$ less than that in KERN group.

One of the aspects that are seen in OA is fascia lateralisation, which takes place from approximately fifth day after carrying out $\mathrm{OA}$. Another aspect of OA is the adhesions between visceral organs, as well as between viscera and abdominal wall, thus forming a "visceral mass". We might deal with lateralisation to some extent with use of CSC, DCS, STAR, and SMAC in VAC, but this is not possible in Kern laparostomy. Adhesions among bowels are inevitable but adhesions between the visceral mass and abdominal wall is preventable in VAC by use of protective membrane, whereas this possibility in Kern laparostomy is only theoretical. This results in a situation that unless the patient with Kern laparostomy is closed

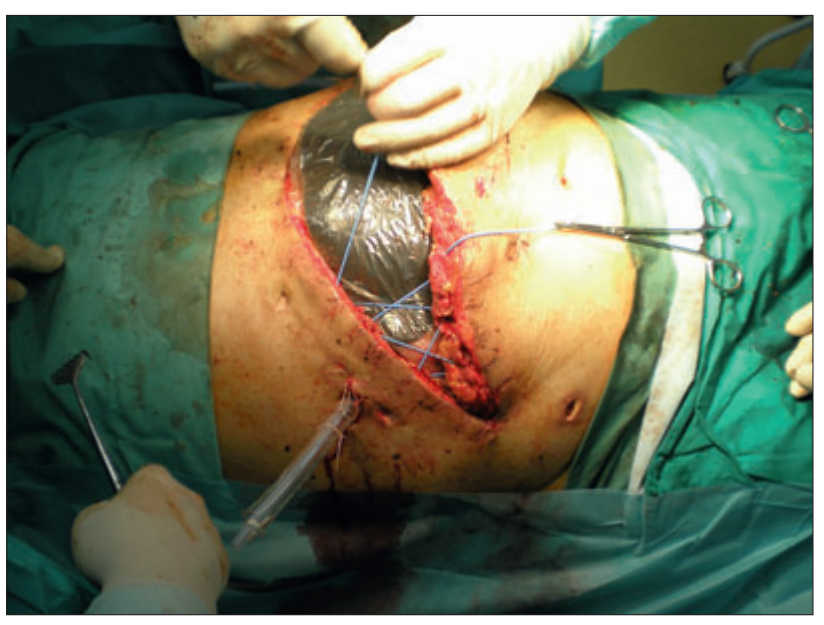

Fig. 3. Use of dynamic compression sutures and VAC in patient with open abdomen. 
$451-454$

in five to seven days, the closure becomes no longer possible and the patient has to survive through overgranulation of the wound with subsequent skin grafting and later incisional hernia repair. In most cases managed with VAC and use of CSC, DCS, STAR, and SMAC, these aspects can be prevented. In our study (Tab. 3), no closure of abdomen in VAC group could be done up to day 7, but eventually we were able to close $88.89 \%$ of patients as opposed to $52.94 \%$ in KERN group.

Fistula is considered the most devastating complication of OA (8) with incidence ranging from $5 \%$ to $75 \%(9,10,11)$. Because fistulas are openings into granulation tissue with no epithelium, they are referred to as being enteroatmospehric. Unfortunately, almost $90 \%$ of these fistulas are of iatrogenic origin. Their incidence is increasing over time; $\mathrm{OA}$ is persisting $(9,10,11)$. Although not significantly stated, there are debates that the use of NPWT increases the risk of fistula formation in OA (12). In our group, there is higher incidence of fistula in VAC group, however without statistical significance (33.3 vs $23.5 \%$ ). Since primary closure is almost impossible, some authors proclaim treatment of fistulas by their resection or proximal stoma (13). Of standard options of treatment, intubation of fistula with Petzer catheter or „floating stoma" do not lead to control of secretion. By using NPWT, the chances grow, but only small fistulas with no eversion of mucosa are prone to primary closure. If this is not possible, enteral contents should be diverted to reduce the septic morbidity of patients (14). Out of couple possibilities, "Fistula VAC" worked the best $(6,15)$. In our study, we were not able to close any fistula or divert fistula content in patients with Kern laparostomy. In VAC group, we successfully closed fistula primarily in one patient $(11.1 \%$ of patients with fistula), while in six patients ( $66.7 \%$ of patients with fistula) we managed to divert the enteral content. Thus, almost $78 \%$ of patients with fistula in OA can be managed successfully by VAC.

All parameters were stratified by BMI and CRP but no statistically significant findings were identified. The study was started also with stratification with PCT (procalcitonine) and APACHE II scoring system but in the retrospective analysis we were not able to identify these in all patients and in absolute values, and thus we discontinued the assessment (Figs 2 and 3).

\section{Conclusion}

The aim of this study was to identify whether the use of intraabdomnial VAC is superior to Kern laparostomy in patients with OA. In this study we proved, that intraabdominal VAC significantly decreases mortality in patients with OA, significantly increases closure of abdominal wall in patients with OA, and significantly increases successful management of enteroatmospheric fistula in open abdomen. No statistically significant findings were identi- fied when stratifying the data with BMI and CRP. Based on this study, intraabdominal use of VAC should replace the use of Kern laparostomy in patients managed by open abdomen.

\section{References}

1. Kern E, Klaue P, Arbogast R. Programmierte Peritoneal-Lavage bei diffuser Peritonitis. Chirurg 1983; 54: 306-310.

2. Olejnik J, Vokurka J., Vician M. Acute necrotizing pancreatitis: intraabdominal vacuum sealing after necrosectomy. Hepatogastroenterology 2008; 82-83: 315-318.

3. Caro A et al. Treatment of the open abdomen with topical negative pressure therapy: a retrospective study of 46 cases. Int Wound J 211; Mar 16 - epub ahead of print www. ncbi.nlm.nih.gov/pubmed/21410648, accessed 9.4.2011

4. Arigon JP et al. Managing the open abdomen with vacuum-assisted closure therapy: retrospective evaluation of 22 patients. J Chir (Paris) 2008; 145 (3): 252-261.

5. Hut'an M Jr, Hut'an M Sr. Sandwich Mesh Abdominal Closure. Bratis Lek Listy 2010; 111 (8): 461-463.

6. Goverman, Yelon, Platz, Singson, Turcinovic. The „Fistula VAC“, a technique for Management of Enterocutaneous Fistulae Arising within the Open Abdomen: Report of 5 cases. J Trauma 2006; 60: 428-431.

7. Björck M, Bruhin A, Cheatham M, Hinck D, Kaplan M, Manca G, Wild T, Windsor A. Classification-important step to improve management of patients with an open abdomen. World J Surg 2009; 33 (6): 1154-1157.

8. Schecter WP, Ivatury RR, Rotondo MF, Hirshberg A. Open abdomen after trauma and abdominal sepsis: a strategy for management. J Am Coll Surg 2006; 203: 390-396.

9. Evenson RA, Fischer JE. Treatment of enteric fistulae in the open abdomen. Chirurg 2006; 77: 594-601.

10. DeCosta A. Making a virtue of necessity: managing the open abdomen. AnZ J Surg 2006; 76: 356-363.

11. Schachtrupp A, Fackeldey V, Klinge u, Hoer J, Tittel A, Toens C, Schumpelick V. Temporary closure of the abdominal wall. Hernia 2002; 6: $155-162$.

12. Shaikh IA, Ballard -Wilson A, Yalamarthi S, Amin AI. Use of topical negative pressure in assisted abdominal closure does not lead to high incidence of enteric fistulae. Colorectal Dis 2010; 12 (9): 931-934.

13. Joyce MR, Dietz DW. Management of complex gastrointestinal fistula. Curr Probl Surg 2006; 46 (5): 384-430.

14. Stremitzer $S$ et al. Successful bridging treatment and healing of enteric fistulae by vacuum-assisted closure (VAC) therapy and targeted drainage in patients with open abdomen. Int J Colorect Dis 2011 Jan 7. [Epub ahead of print] http://www.ncbi.nlm.nih.gov/pubmed/21212963

15. Khoury, Kaufman, Hirshberg. Improved Control of Exposed Fistula in the Open Abdomen. J Am Coll Surg 2008; 206 (2): 397-398.

Received August 24, 2011. Accepted January 23, 2013. 